\title{
SOURCES OF NOISE IN HIGH-RESOLUTION TRACKING WITH SCINTILLATING FIBRES
}

\author{
WA 84 Collaboration
}

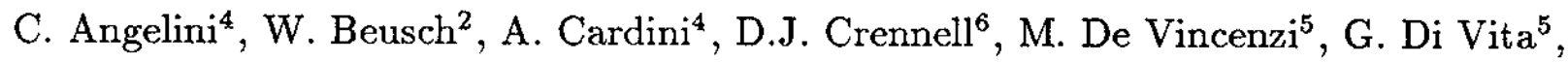
A. Duane ${ }^{3}$, J.P. Fabre ${ }^{2}$, V. Flaminio ${ }^{4}$, A. Frenkel ${ }^{5}$, T. Gys ${ }^{2}$, K. Harrison ${ }^{3}$, E. Lamanna ${ }^{5}$, H. Leutz ${ }^{2}$, G. Martellotti ${ }^{5}$, J.G. McEwen ${ }^{7}$, D.R.O. Morrison ${ }^{2}$, G. Penso ${ }^{5}$, S. Petrera ${ }^{5}$, C. Roda ${ }^{4}$, A. Sciubba ${ }^{5}$, E. Vicari ${ }^{2}$, D.M. Websdale ${ }^{3}$, G. Wilquet ${ }^{1}$ (Presented by G.Penso)

\begin{abstract}
Noise generated in the WA84 scintillating fibre vertex detector has been studied both experimentally and theoretically. The different sources of noise have been identified, and their relative contributions determined. Methods of noise reduction are considered.
\end{abstract}

Talk given at the $4^{\text {th }}$ Pisa Meeting on Advanced Detectors.

Frontier Detectors for Frontier Physics.

La Biodola - Isola d'Elba - May 1989

(To be published in Nucl. Instr. and Meth.)

${ }^{1}$ Inter-University Institute for High Energy Physics (ULB-VUB), Brussels, Belgium.

2 CERN, Geneva, Switzerland.

${ }^{3}$ Imperial College, London, UK.

4 Università di Pisa and INFN, Pisa, Italy.

5 Università di Roma "La Sapienza" and INFN, Rome, Italy.

${ }^{6}$ Rutherford Appleton Laboratory, Didcot, UK.

${ }^{7}$ University of Southampton, UK. 


\section{Introduction}

The newly developed plastic scintillating fibres (SCIFI) of diameter $\leq 30 \mu \mathrm{m}$, combined with the advances in optoelectronic technology, now allow the building of a high-resolution, fast, radiation hard, tracking device. Such a detector is being developed for the WA84 experiment [1] at CERN. The aim of this experiment is to study the hadroproduction and decay of beauty particles and in particular to measure the charged and neutral B-meson life-times separately.

The detector will be placed in a high intensity $\left(\simeq 10^{6}\right.$ particles per second $)$ $350 \mathrm{GeV} / \mathrm{c} \pi^{-}$beam at the CERN Super Proton Synchrotron. The events where a $\mathrm{B} \overline{\mathrm{B}}$ pair is produced, are expected to be characterized by a high track multiplicity, and by up to five vertices, one from the primary interaction and the others from $B, \bar{B}, D$, and $\bar{D}$ decays. In order to reconstruct such events, high resolution $(\leq 25 \mu \mathrm{m})$ particle tracking in the target is needed. Furthermore, spurious hits, i.e. hits away from the particle tracks, should be as few as possible. These hits, which give rise to noise on the event picture, are due to many different phenomena which take place either in the target or in the optoelectronic chain.

In this paper we present a quantitative analysis of the most important phenomena that contribute to the image noise in our vertex detector. These phenomena have been studied experimentally and simulated by the Monte Carlo method. The possibility of reducing or even eliminating some of them has also been investigated.

\section{The experimental set-up}

The experimental set-up of the WA84 experiment has been described in detail elsewhere $[2,3,4]$. A vertex detector is used in conjunction with the Omega spectrometer [5]. The vertex detector (fig. 1) consists of a target which is a coherent bundle of scintillating microfibres ( $\sim 30 \mu \mathrm{m}$ width) parallel to the beam line, coupled to a series of five image-intensifiers (I.I.\#1 to \#5, fig. 1) by a bent taper which magnifies the image by a factor $\sim 3.4$. The first two image-intensifiers are inside the magnetic field $(1.8 \mathrm{~T})$ of the Omega spectrometer and therefore must be of the proximity-focused type $\left.(\text { Proxifier })^{\star}\right)$ which can operate with a magnetic field perpendicular to their faceplates. An image guide brings the light out of the magnetic field to the last three imageintensifiers which are electrostatically-focused (I.I.\# $3^{\star \star}$ ) and I.I.\# $5^{\times)}$) or proximityfocused with a microchannel plate electron multiplier (I.I.\# $4^{\times x}$ ). The latter is gated by the first level trigger. This allows us to record on the charge-coupled device (CCD) $\dagger$ ) placed at the end of the optoelectronic chain, only events due to beam particles which interact in the target.

^) Proxitronic GmbH,6140 Bensheim, Fed. Rep. Germany.

**) Varo, Inc., Electron Device Division, Garland, Texas, USA. Ref. 25/46.

$\times$ ) Delft Electronische Producten, Postbus 60, 9300 AB Roden, The Netherlands.

$\times x$ ) ITT F4113: PO Box 3700, Fort Wayne, Indiana 46801, USA.

$\dagger$ †) Thomson-CSF TH 7864, BP 305, 92102 Boulogne-Billancourt, Cedex, France. 


\section{Sources of noise on event pictures}

A typical picture of a $200 \mathrm{GeV}$ hadron interaction is shown in fig. 2. The beam particle is perpendicular to the figure so it appears as a spot at the interaction point. The secondary particles coming from this point are clearly seen as a sequence of well aligned hits. Apart from these, many other hits are spread over the picture. This is the image noise we are considering. It can originate in different parts of the set-up:

- optical cross-talk in the target;

- electron backscattering in the image-intensifiers;

- electronic noise in the CCD read-out.

Beyond these instrumental effects $\delta$-rays also contribute to the noise.

In the next sections the different sources of noise will be considered separately and their contributions to the overall image noise will be evaluated.

\section{Optical noise in the target}

Before analysing the sources of optical noise in the target, let us recall some properties of scintillators in connection with microfibre detectors. In a high resolution scintillating fibre target the light emission must be "local" in the sense that it must take place at distances from the ionizing particle trajectory which are short compared to the fibre diameter. This condition is normally fulfilled for inorganic scintillators (like the GS1 scintillating glass). In organic scintillators the opacity of the base material to its own scintillation light forces the introduction of a wavelength shifter (WLS) which absorbs this primary light and reemits at a wavelength where the material is more transparent and standard photocathodes are more efficient. For usual (low) WLS concentrations this secondary light emission takes place within some hundreds of microns from the trajectory of the ionizing particle. Therefore organic scintillators with a standard composition are not suitable for high-resolution tracking with scintillating microfibres (diameter $\leq 30 \mu \mathrm{m}$ ). To restore the locality of the light emission it is necessary to increase the WLS concentration so as to decrease the mean free path of the primary light before being waveshifted. The high concentration needed to obtain the desired effect favours those WLS (like PMP [6]) which have a large Stokes' shift, resulting in low self-absorption of the secondary light. In conclusion high-resolution tracking with scintillating fibres can be performed using either inorganic scintillators or organic ones but with a WLS concentration much higher than normally used.

Let us now consider the problem of optical noise in the target. By optical noise we mean the light which at the end of the target emerges from fibres different from those in which it was produced by an ionizing particle. We should remember that the trapping efficiency of a fibre is of the order of a few percent and therefore most of the scintillation light escapes the fibre where it was produced and becomes a potential source of to noise.

Optical noise in the target may have various origins. The overlap of emission and absorption spectra of the final light emitter allows self-absorption of the light which is initially untrapped and subsequent isotropic reemission and trapping in a fibre different from the initial one. 
A second source of noise is possibly due to imperfections of the core-cladding interface which can cause light scattering and subsequent trapping in any fibre of the target. Careful manufacture of the target can reduce this effect.

A third source is due to light trapped in the whole target which behaves like a single fibre with an air cladding and therefore with a numerical aperture much larger than that of a single microfibre. This effect can be eliminated by an appropriate black coating of the external surface of the target.

Another source consists of light rays being trapped in subsets of fibres inside the target over distances of the order of the target length. This phenomenon has been studied in detail with a Monte Carlo simulation, which will be described in the next section.

A method of avoiding these phenomena is to surround each microfibre with an extra mural absorber (EMA) coating. Increasing the absorption efficiency of the EMA will decrease the observed noise. It should be noted that for the fibre diameters we are considering the EMA thickness cannot be much more than $\sim 1 \mu \mathrm{m}$, otherwise the packing fraction of the fibre (core cross-section / fibre cross-section ) will be appreciably affected. The problem is therefore to find an EMA material which absorbs a non-negligible fraction of scintillation light in a thickness of the order of $1 \mu \mathrm{m}$, and which meets other mechanical, thermal and chemical properties needed in the SCIFI manufacturing process. This problem has been solved for glass fibres but not yet for plastic fibres. If no EMA is present the problem of cross-talk between fibres may become relevant.

\section{Monte Carlo tracking of light rays in a microfibre target}

A complete Monte Carlo simulation of light emission and propagation, based on simple geometrical optics, has been set up. Two possible shapes of microfibre crosssection have been considered which are those generally encountered, namely hexagonal $\$$ ) and square. In the following discussion we will first consider hexagonal fibres. Perfectly smooth core and cladding surfaces are supposed. Core and cladding dimensions and refractive indices $\left(n_{\text {core }}, n_{\text {clad }}\right)$, as well as target dimensions are given as input parameters. An exponential absorption of light in the core material has been assumed. In order to reproduce the difference between the light attenuation length in bulk $\left(\lambda_{\text {bulk }}\right)$ and in fibres $\left(\lambda_{\text {fibre }}\right)$ of the same material and the increase of $\lambda_{\text {fibre }}$ with the fibre diameter [7], the total reflection coefficient $(\mathrm{R})$ at the core-cladding interface has been taken to be less than 1.

In the following we present some Monte Carlo results for two target configurations, built of $30 \mu \mathrm{m}$ hexagonal fibres with a $3 \mu \mathrm{m}$ thick cladding and no EMA: i) one in the form of a cylinder $50 \mathrm{~mm}$ long, $5 \mathrm{~mm}$ in diameter, black-coated with fully absorbing paint on its outer surface; ii) one in the form of a parallelepiped of the same length and $5 \times 5 \mathrm{~mm}^{2}$ cross-section. The latter target is built from black-coated fibre bundles having a square cross-section of $1 \times 1 \mathrm{~mm}^{2}$. In both cases the non-readout end is

†) Plastic fibres which are initially circular in cross-section, become hexagonal during the target manufacturing process. 
supposed to be equipped with an $80 \%$ efficient mirror. We have assumed $n_{\text {core }}=1.59$, $n_{\text {clad }}=1.46, \lambda_{\text {bulk }}=150 \mathrm{~cm}, \mathrm{R}=0.9997$. These numbers lead to a value for $\lambda_{\text {fibre }}$ of $\sim 20 \mathrm{~cm}$ at short distances, which is representative of a typical $30 \mu \mathrm{m}$ plastic fibre [8].

Scintillation light is generated isotropically and uniformly in a cross-section of the central fibre of the target, at any given longitudinal position along this fibre. Every light ray is then followed along the target to its viewing end. Light arriving at the black coating is lost. The light emerging from the end of the target is divided into two categories: that coming out of the fibre in which it was generated, and that coming out of any other fibre. The former will be referred to as signal, the latter as noise. In fig. 3 we show, as an example, the projection on a plane perpendicular to the target axis of a light ray which contributes to noise, travelling along the target from its starting point to its exit point.

In fig. 4a) and b) we show the angular distribution, with respect to the target axis, of the signal and noise respectively, when light is generated near the end opposite to the readout. As expected the angular distribution for the noise is broader than for the signal. The peak at very forward angles $(\cos \theta \geq 0.8)$ in the angular distribution of the noise is due to light rays emerging from the cladding of the fibres, while that around $\cos \theta \simeq 0.7$ results from noise emerging from the cores. These features are qualitatively confirmed by a simple experimental measurement that will be described in the next section.

In fig. 5 the noise-to-signal intensity ratio is plotted versus the longitudinal distance $(z)$ between the light emission point and the readout window. It shows that this source of noise cannot be neglected, especially in short targets. The behaviour of the noise-to-signal ratio in fig. 5 is mainly due to the presence of the black coating on the target or the fibre bundles. Reducing the distance from the emission point to the nearest absorbing black surface, as in the second configuration, drastically reduces the noise level.

In fig. 6 the behaviour of the noise level per unit area on the readout window is shown as a function of the distance between the exit point of the light and the target axis. It shows that the noise decreases rapidly with the distance from the target axis but extends to the nearest absorbing black surface.

In conclusion, even if no EMA is present on each microfibre, the amount of noise emerging from the readout window is expected to be significantly reduced by assembling a target from many black-coated fibre bundles to obtain the desired dimensions. This result has been confirmed experimentally as we will show in section 9 .

In the case of square microfibres, light escaping from a fibre moves away more rapidly than for hexagonal ones, quickly reaching the nearest absorbing surface. Therefore there is a steep decrease of the level of noise with the longitudinal distance between the emission point and the readout window. For example, in a target similar to the second configuration already described, but with $30 \times 30 \mu \mathrm{m}^{2}$ square microfibres, the Monte Carlo shows that at a longitudinal distance of $5 \mathrm{~mm}$, the noise is less than $5 \%$ of the signal. In this respect, targets made of square microfibres are much less noisy than those of hexagonal ones. 


\section{Experimental angular distribution of light emitted by the target}

A simple set-up (fig. 7) has been used to measure the angular distribution of signal and noise light emitted by the target. A UV lamp $(\lambda=254 \mathrm{~nm})$ illuminates one of the end windows of the target through a $1 \mathrm{~mm}$ diaphragm, generating scintillation light in the corresponding microfibres. At the other end of the target a similar diaphragm or its complement (i.e. a black absorbing spot) is aligned with the first diaphragm. They allow the selection of either the light trapped in the fibre in which it was generated (signal) or its complementary light which arrives at the exit of the target (noise). This experimental situation is similar to that simulated by the Monte Carlo, whose results were given in fig. 4. The light yield is obtained by measuring the anode current of a photomultiplier tube RCA 8850 Quantacon. By varying the distance ( $d$, fig. 7) between the photocathode of this tube and the target, we measure the integral angular distribution for signal and noise, from which we deduce the differential angular distribution, shown respectively in fig. $8 \mathrm{a}$ ) and b). The angular distribution of the signal appears much more peaked in the forward direction than that of the noise. This confirms the behaviour predicted by the Monte Carlo (fig. 4a). Despite the roughness of the experimental method, the two-peak structure predicted by the Monte Carlo in the angular distribution of the noise (fig. 4b), is also seen in the experimental results (fig. 8b), but much more broadened. This may be due to the non-ideal geometrical and optical characteristics of the real target and to the presence of noise sources other than that simulated by the Monte Carlo.

It is however important to note that the quite different angular distributions of the signal and noise allow a significant reduction in the noise-to-signal ratio, at the cost of a minor signal attenuation, by accepting only the light emitted by the target in a limited angular region with respect to its axis.

\section{Noise from Image-Intensifiers}

The electrons emitted by the photocathode of an image-intensifier are accelerated by an electrostatic field to a phosphor screen where, sometimes, backscattering may occur. In the WA84 vertex detector, this phenomenon is an important source of widespread noise, especially in I.I.\#1, if no magnetic field is present. The scattered electrons may be emitted at any angle, with an energy lower than or equal to their incident energy. They then fall again on the phosphor at different points, giving rise to noise on the intensified image.

This phenomenon can, in principle, occur in any image-intensifier of the optoelectronic chain, but its contribution to the final image noise comes mainly from the first one. In fact, for a minimum ionizing particle, the light intensity which comes out of the target is so low that most of the time a hit on the track image is due to a single photoelectron in the first image-intensifier. If this electron backscatters it will be lost as a "signal hit", reappearing elsewhere on the image, as a "noise hit". The light intensity of a "noise hit" will therefore be lower or equal to that of a "signal hit", depending on the energy lost by the electron in the scattering process. The situation is clearly different in the downstream image intensifiers where backscattered electrons produce 
"noise hits" of a much lower intensity than that of the "signal hits", already intensified by the previous stages. By placing an off-line threshold on the charge collected by the CCD pixels, the noise due to electron backscattering in the first image intensifier can be somewhat reduced, while that produced in the downstream image intensifiers becomes negligible. The effect of this threshold on the event of fig. 2a) is shown in fig. $2 b$ ). In addition to the background reduction, a better spatial resolution is also achieved around the interaction point. Nevertheless, this threshold cannot be too high or hits on a minimum ionizing particle track are lost.

For a proximity-focused image-intensifier it is easy to show that, if no magnetic field is present, the maximum distance $\left(r_{\max }\right)$ that an electron can reach from the scattering point, on the phosphor screen, is equal to twice the distance between the photocathode and the phosphor. In our case $r_{\max }=10 \mathrm{~mm}$, to be compared with the $12.5 \mathrm{~mm}$ radius of the sensitive zone of the phosphor. Therefore, practically all the image surface can be affected by this type of noise.

We have performed a quantitative measurement of this phenomenon in our I.I.\#1, using a modified version of the set-up of fig. 1. The target, the bent taper and the I.I.\#2 were removed while the I.I.\#1 under investigation was placed in direct contact with the image guide followed by the rest of the optoelectronic chain. A pulsed light spot ( $1 \mathrm{~mm}$ diameter) was formed on its photocathode, in an off-centre position. This allows the observation of the light distribution on the phosphor at larger distances from the image spot. The gate $(\sim 1 \mu \mathrm{s})$ of I.I.\#4 was opened in coincidence with the light pulse.

In fig. 9 a superposition of several hundred images obtained on the CCD is shown at the scale of the phosphor screen of I.I.\#1. Around the image spot a circular zone is clearly seen where the noise is more intense than in the rest of the frame. This corresponds to the kinematically allowed zone where backscattered electrons can fall on the phosphor $\left(r \leq r_{\max }\right)$.

In fig. 10 we show the light intensity per unit area emitted by the phosphor as a function of the distance from the centre of the image spot. A fast decrease around $\mathrm{r} \simeq 8 \mathrm{~mm}$ is evident. Below this distance the surface density of the noise is almost flat. To account for this behaviour a simple Monte Carlo simulation of the backscattering process has been made. A triangular energy distribution [9] of the scattered electrons has been assumed, peaked at $\alpha \mathrm{E}$ where $0 \leq \alpha \leq 1$ and $\mathrm{E} \simeq 28 \mathrm{keV}$ is the incident electron energy. The angular distribution of the scattered electrons was assumed to be of the form $\mathrm{dN} / \mathrm{d}(\cos \theta) \propto \cos ^{\mathrm{n}} \theta$, where $\theta$ is measured with respect to the normal to the phosphor plane. No correlation between angle and energy of the scattered electrons has been considered. The backscattering probability was left as a free parameter. With these assumptions the experimental results of fig. 10 can be well reproduced for $\mathrm{r} \leq 10$ $\mathrm{mm}$. As an example we show on that figure the curve obtained for $\alpha=0.6$ and $\mathbf{n}=5$, but equally good fits can be obtained with somewhat different values of these two parameters. For $r \geq 10 \mathrm{~mm}$ we observe in fig. 10 a low-level noise which of course is not predicted by this simple Monte Carlo simulation. It may be due to double backscattering of the electrons or light diffusion in I.I.\#1. 
The noise-to-signal ratio, defined as the ratio between the total light yield in the regions $0.5 \leq \mathrm{r} \leq 10 \mathrm{~mm}$ and $\mathrm{r} \leq 0.5 \mathrm{~mm}$, is found to be $\simeq 12 \%$. Assuming the above-mentioned energy distribution, with $\alpha$ and $\mathrm{n}$ values chosen to fit the experimental distribution of fig. 10 , leads to a backscattering probability around $20 \%$. This value is somewhat different from the backscattering probability for the $\mathrm{Y}_{3} \mathrm{Al}_{5} \mathrm{O}_{12}$ phosphor used, which is $\sim 13 \%$, almost independent of electron energy in the range $10-100 \mathrm{keV}$ [10]. A more refined Monte Carlo simulation, taking into account correlations between energy and angle of backscattered electrons [11] would be expected to give a better agreement. Nevertheless, the backscattering probability found is of the correct order of magnitude.

On an event image, the distribution of the noise due to electron backscattering in I.I.\#1 is strongly affected by the presence of a magnetic field. In the WA84 set-up the $1.8 \mathrm{~T}$ field reduces $\mathrm{r}_{\max }$ to $\sim 0.6 \mathrm{~mm}$ corresponding to $\sim 0.2 \mathrm{~mm}$ at the target surface. The effect of the magnetic field is therefore to concentrate the noise due to electron backscattering near the particle track images. Some pictures of beam tracks taken in the Omega spectrometer, with and without magnetic field, confirm this effect, as will be described in section 9 .

Finally we note that gating $(\sim 1 \mu$ s) the I.I.\#4 eliminates noise due to thermal electrons emitted by the photocathode of the upstream image intensifiers.

\section{Noise from CCD}

The CCD and its electronic read out could, in principle, be a source of noise. In practice the thermal noise of the CCD becomes completely negligible if a fast clear is applied at a rate of the order of some tens of $\mathrm{Hz}$ or higher. Furthermore a hardware threshold is applied on the analog-to-digital converter which measures the charge collected by the CCD pixels. At the end of the optoelectronic chain the intensity of the amplified light is so high that it allows the elimination of the electronic noise of the preamplifier without losing hits on the picture. In conclusion the CCD and its readout system do not contribute to the observed noise.

\section{Noise measurements with particle tracks in the full vertex detector}

We have collected many thousands of events with the fully mounted WA84 vertex detector, with different targets and in different experimental conditions. The trigger selects beam particles crossing the target at an angle of $20^{\circ}$ with respect to the fibre axes. A typical picture obtained with this configuration is shown in fig. 11. Hits on the track are fitted with a straight line ( $\mathrm{TT}^{\prime}$, fig. 11). Then many hundred of tracks are superimposed and all the pixels, weighted with their pulse heights, are projected onto an axis perpendicular to the $\mathrm{TT}^{\prime}$ line ( $x$ axis, fig. 11) with the origin at their intersection. In fig. 12 we show the projected pulse height spectra obtained with this method, for three different plastic SCIFI targets ${ }^{\star}$. Each fibre has an almost hexagonal cross-section, but no EMA. The signal, which corresponds to the central bin, is defined

$\star$ The fibre cores were either SCSN-81T(0.5) or PMP doped polystyrene, the two being found to be equivalent for the study of noise. 
to be the pulse height within $3 \sigma$ of the track, where $\sigma \simeq 20 \mu \mathrm{m}$ is the r.m.s. track residual. The noise is the pulse height integrated over all the $x$ axis except the central bin. The upper histogram (solid line) is for a target without black coating on its outer surface. The intermediate histogram (dotted line) is for the same target with an outer black coating, while the lower one (dashed line) is obtained for a target assembled from $0.5 \times 0.5 \mathrm{~mm}^{2}$ fibre bundles, each bundle being black-coated before assembling. For these three targets, the measured percentage contributions from all forms of noise to the total light detected are $\sim 48 \%, \sim 40 \%$ and $\sim 25 \%$ respectively.

In fig. 13 we show the pulse height distribution, projected onto the $x$ axis, without (solid line) and with (dashed line) magnetic field $(1.8 \mathrm{~T})$ perpendicular to the face plates of I.I.\#1 and \#2 (fig. 1). These spectra have been obtained with a GS1 glass SCIFI target. Each fibre has a square cross-section and is coated with a partially absorbing EMA, so the noise level is comparable to that of a plastic SCIFI made of black-coated fibre bundles. The magnetic field reduces the noise at larger distances from the track and increases it at shorter distances. This is as expected because, as already noted, the magnetic field concentrates most of the backscattered electrons in a small region around the scattering points, which are along the track image. That noise which is still present at large $(\geq 0.6 \mathrm{~mm}) x$ values when magnetic field is on (dashed line of fig. 13) is therefore due to sources other than electron backscattering in image intensifiers.

\section{Delta rays}

The $\delta$-rays generated by particles crossing the target have, most of the time, insufficient energy to produce a clearly visible track. Therefore they contribute to the total noise. A theoretical estimate of that contribution has been performed by a Monte Carlo simulation of $\delta$-ray production in conditions corresponding to fig. 11 . The average energy per unit volume released in the target by the particle and the associated $\delta$-rays has been computed as a function of the distance from the particle track. This distribution was projected onto the $x$ axis as defined in fig. 11 . The resulting pulse height distribution is shown in fig. 14. Assuming that, within a distance $\Delta x= \pm 3 \sigma \simeq \pm 60 \mu \mathrm{m}$ from the track, hits due to $\delta$-rays cannot be distinguished from those due to the particle, the noise-to-signal ratio, defined as the pulse height on frame due to $\delta$-rays divided by that due to the generating track, is $\sim 11 \%$.

\section{Conclusions}

Images of particle tracks in various SCIFI targets have been recorded on a CCD using the optoelectronic chain developed for the WA84 experiment. Tracks may be clearly seen but with a large amount of accompanying noise. For a given target, this noise is proportional to the light intensity along the tracks.

Three sources of noise have been identified: $\delta$-rays in the target, backscattering of electrons in image-intensifiers and optical cross-talk between fibres. The contribution to the total noise from $\delta$-rays has been calculated to be $\sim 11 \%$ of the signal, independent of the target configuration. Noise-to-signal ratio for the image intensifiers alone amounts to $\sim 12 \%$, if no magnetic field is present. With a $1.8 \mathrm{~T}$ magnetic field perpendicular to 
the faceplates of the Proxifiers, this noise is concentrated near the track image, with a maximum range of $\sim 0.2 \mathrm{~mm}$ as measured at the target surface. Increasing the optical magnification between the target and the first Proxifier decreases this maximum range so that backscattered electrons are no longer seen as noise. With a target constructed from $30 \mu \mathrm{m}$ hexagonal plastic fibres with no EMA and no black-coating, and in the absence of magnetic field, the experimentally measured noise is $\sim 48 \%$ of the total detected light. This is the sum of $\sim 10 \%$ from $\delta$-rays, $\sim 32 \%$ attributed to optical cross-talk and $\sim 6 \%$ due to electron backscattering in the image intensifiers. Substantial reductions in the optical noise are obtained by the use of light absorbers. When the outer surface of the target is black-coated, the experimentally measured noise is $\sim 40 \%$ of the total light, comprising $\sim 10 \%$ from $\delta$-rays, $\sim 23 \%$ from cross-talk and $\sim 7 \%$ from electron backscattering. For a target assembled from $0.5 \times 0.5 \mathrm{~mm}^{2}$ black-coated fibre bundles, the measured noise is $\sim 25 \%$ of the detected light, given by $\sim 10 \%$ from $\delta$-rays, $\sim 6 \%$ from cross-talk and $\sim 9 \%$ from the image intensifiers. An alternative means of reducing the optical noise is to accept only light emerging from the target at relatively small angles with respect to the fibre axes. This can be done, for example, by replacing the bent taper by a lens-mirror system which has the advantage of being radiation-hard.

Improvements in SCIFI manufacturing could eventually enable a high efficiency EMA to be placed on individual plastic fibres. Combined with a better quality corecladding interface and with the newly developed wavelength shifters, such as PMP, this would avoid any cross-talk.

In conclusion, all forms of noise can in principle be eliminated, with the exception of $\delta$-rays.

\section{Acknowledgements}

We are pleased to acknowledge the continued support of Drs. H. Wenninger and J.C. Gouache, EF Division, CERN, during the development of this work. We are also grateful to the engineers and technicians of the EF Instrumentation Group, in particular to J. Dupont, J. Dupraz, P. Nappey, D. Piedigrossi, and S. Reynaud for their invaluable help, and to M. Punturo for performing some of the numerical calculations. 


\section{REFERENCES}

[1] W. Beusch et al., CERN/SPSC 87-2, P226 (1987).

[2] C. Angelini et al., Nucl. Instr. and Meth. A277 (1989) 132.

[3] C. Roda, Thesis, University of Pisa. CERN/EF/INSTR 89-1 (May 1989).

[4] C. Angelini et al., preprint CERN-EP/89-119 (1989), presented by K. Harrison at this Conference.

[5] W. Beusch et al., CERN/SPSC 77-70, SPSC/T-17 (1977).

[6] P. Destruel et al., Nucl. Instr. and Meth. A276 (1989) 69.

[7] C. D'Ambrosio et al., CERN-EP/89-44 (March 1989); Proc. of the Workshop on Scintillating Fibre Development for SSC. Fermilab, Batavia, USA. November 1988.

[8] C. Angelini et al., CERN-EP/89-112 (September 1989) to be published in Nucl. Instr. and Meth.

[9] I. R. McDonald et al., J. Phys. D: Appl. Phys. 4 (1971) 1210.

[10] T. Tabata et al., Nucl. Instr. and Meth. 94 (1971) 509.

[11] M. Fouassier et al., Acta Electronica 20 (1977) 369. 


\section{FIGURE CAPTIONS}

Fig. 1: Schematic view of the vertex detector of the WA84 experiment. The target, the bent taper and the first two image intensifiers are placed in the magnetic field $(1.8 \mathrm{~T})$ of the Omega spectrometer.

Fig. 2: a) Image of a $200 \mathrm{GeV}$ hadron interaction in the target, as seen by the vertex detector. b) The same event as in a) but with an off-line threshold on the charge collected by the CCD pixels.

Fig. 3: Example of light trapping in subsets of hexagonal fibres inside the target. The line, obtained by a Monte Carlo simulation, represents a light ray as seen from the readout window of the target. Light is generated at $\mathrm{A}$ and exits at $\mathrm{B}$. Some fibres of the target are drawn.

Fig. 4: Angular distribution with respect to the target axis of the scintillation light emitted by the target, as obtained by a Monte Carlo simulation. Signal light (a) and noise light (b) are shown separately.

Fig. 5: Noise-to-signal ratio, calculated by a Monte Carlo simulation, as a function of the longitudinal distance between the point where light is generated and the readout window of the target. Solid line: cylindrical target, $5 \mathrm{~mm}$ in diameter, blackcoated on the outside. Dashed line: target assembled from $1 \times 1 \mathrm{~mm}^{2}$ black-coated bundles.

Fig. 6: Density per unit area of the noise on the readout window of the target, calculated by a Monte Carlo simulation, as a function of the distance between the exit point of the light and the target axis. Solid line: cylindrical target, $5 \mathrm{~mm}$ in diameter, black-coated on the outside. Dashed line: target assembled from $1 \times 1 \mathrm{~mm}^{2}$ blackcoated bundles. The two histograms are normalized to 1 in the first bin.

Fig. 7: Schematic view of the set-up used to measure the angular distribution of signal (upper) and noise (lower) light from scintillation in the target.

Fig. 8: Experimental angular distribution of the scintillation light emitted by the target, as obtained with the set-up of fig. 7. a) signal light; b) noise light. Error bars are shown for three points only.

Fig. 9: Superposition of many images recorded on the CCD of a light spot of $\sim 1 \mathrm{~mm}$ in diameter, projected on the photocathode of I.I.\#1. Distances are measured at the phosphor surface. The dark area with a circular outline is due to electrons backscattered by the phosphor from the central spot.

Fig. 10: Surface density of the light emitted by the phosphor, as shown in fig 9, versus the distance from the centre of the light spot. The dotted line is a fit to the data below $r_{\max }=10 \mathrm{~mm}$, obtained by a Monte Carlo simulation of the scattering process.

Fig. 11: Image of a minimum ionizing particle crossing the target at an angle of $20^{\circ}$ with respect to the target axis. The straight line $\mathrm{TT}^{\prime}$ is a fit to the track. The $x$ axis is perpendicular to the $\mathrm{TT}^{\prime}$ line, with the origin at their intersection. Distances are measured at the target surface. 
Fig. 12: Experimental distribution of the pulse height of the CCD pixels, projected onto the $x$ axis defined as in fig. 11. The three histograms, normalized to the same value in the central bin, are for three different plastic targets. Solid line: target without any blackcoating. Dotted line: target with a black-coating on outer surface. Dashed line: target assembled from $0.5 \times 0.5 \mathrm{~mm}^{2}$ black-coated bundles.

Fig. 13: Experimental distribution of the pulse height of the CCD pixels, projected onto the $x$ axis defined as in fig. 11 for a scintillating glass target without (solid line) and with (dashed line) a $1.8 \mathrm{~T}$ magnetic field perpendicular to the faceplates of I.I.\#1 and \#2. The two distributions are normalized to the same integral.

Fig. 14: Calculated pulse height distribution, projected onto the $x$ axis as defined as in fig 11 , due to $\delta$-rays. 


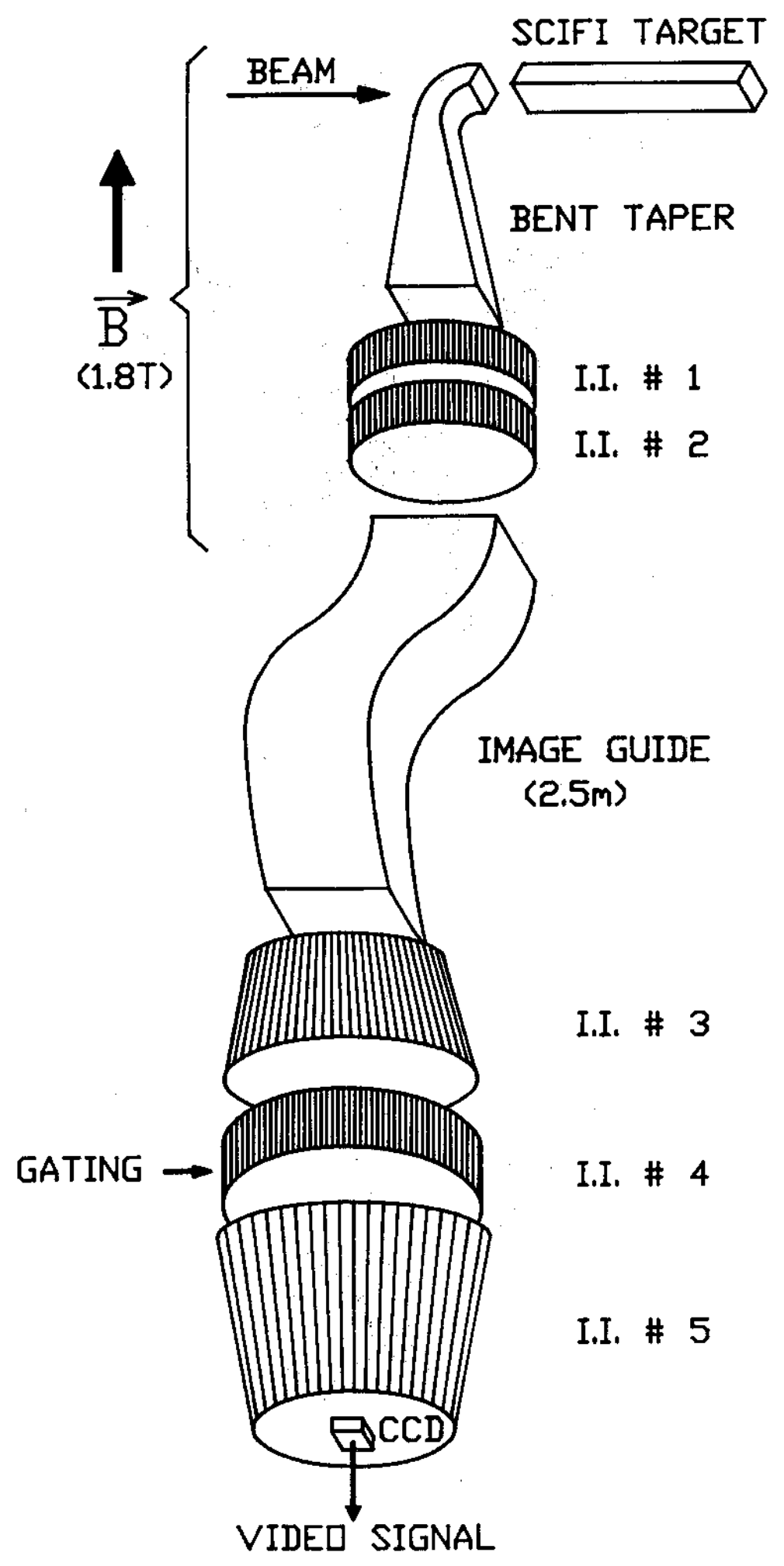

Fig. 1 

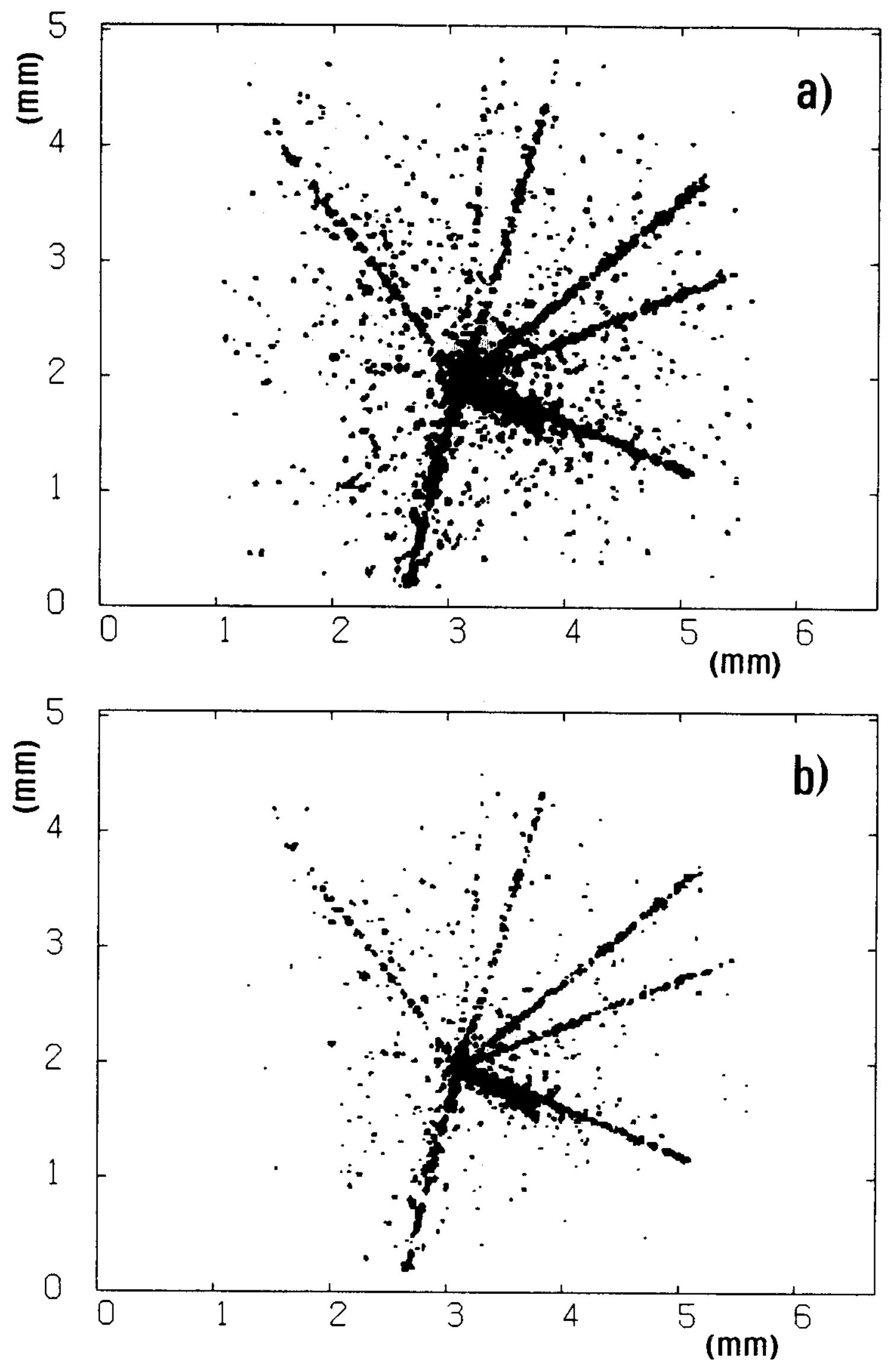

Fig. 2 


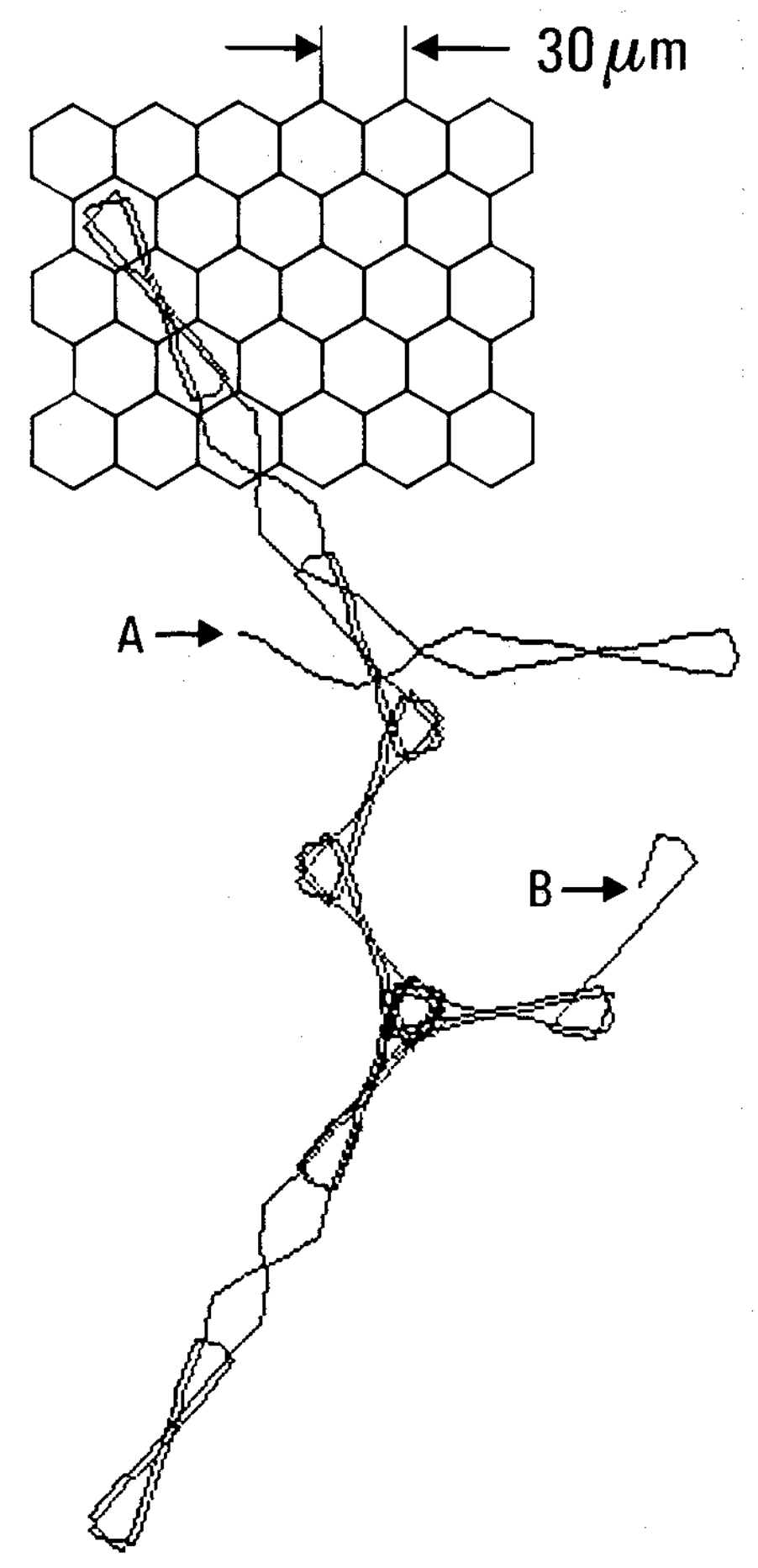

Fig. 3 

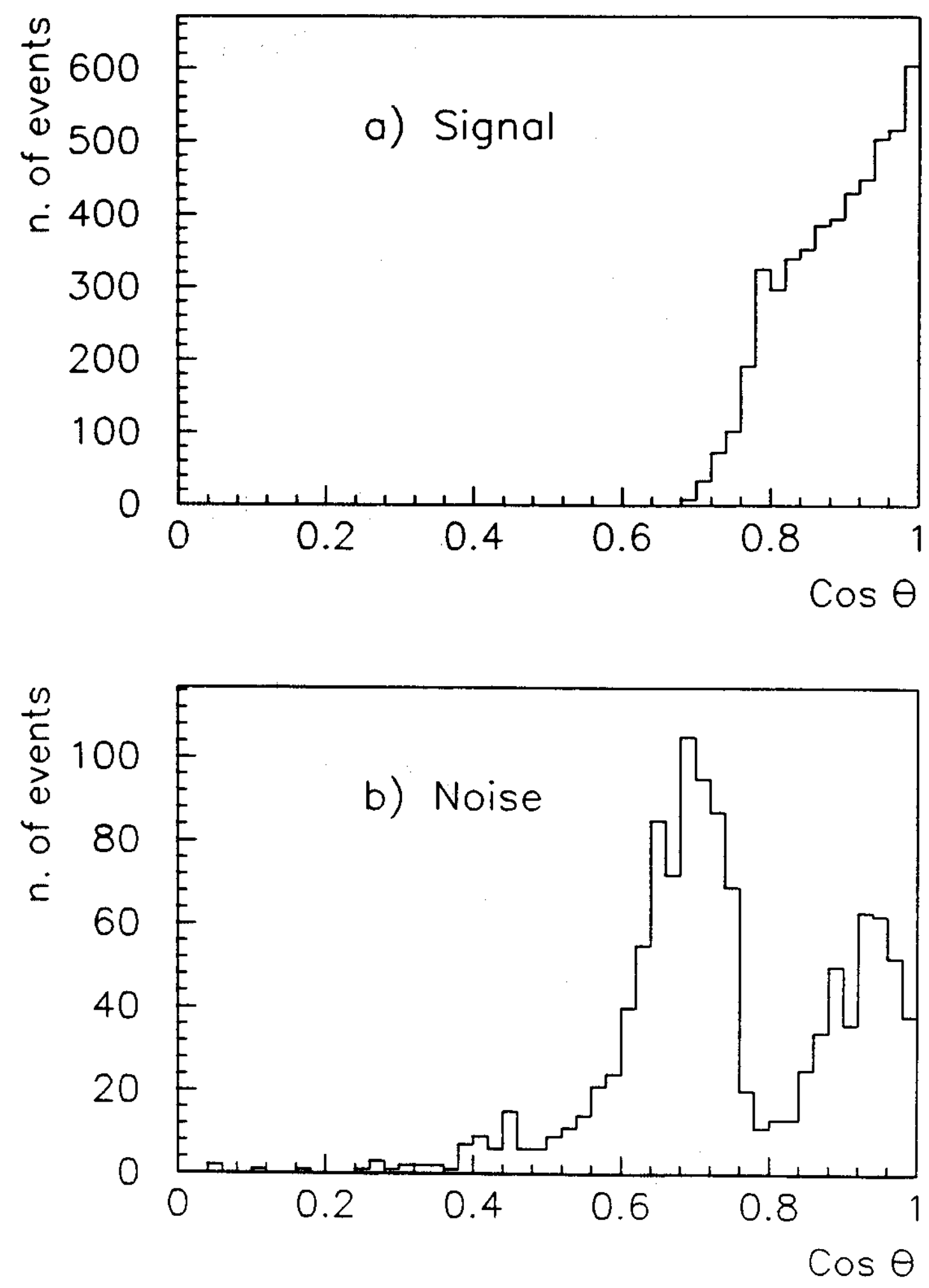

Fig. 4 


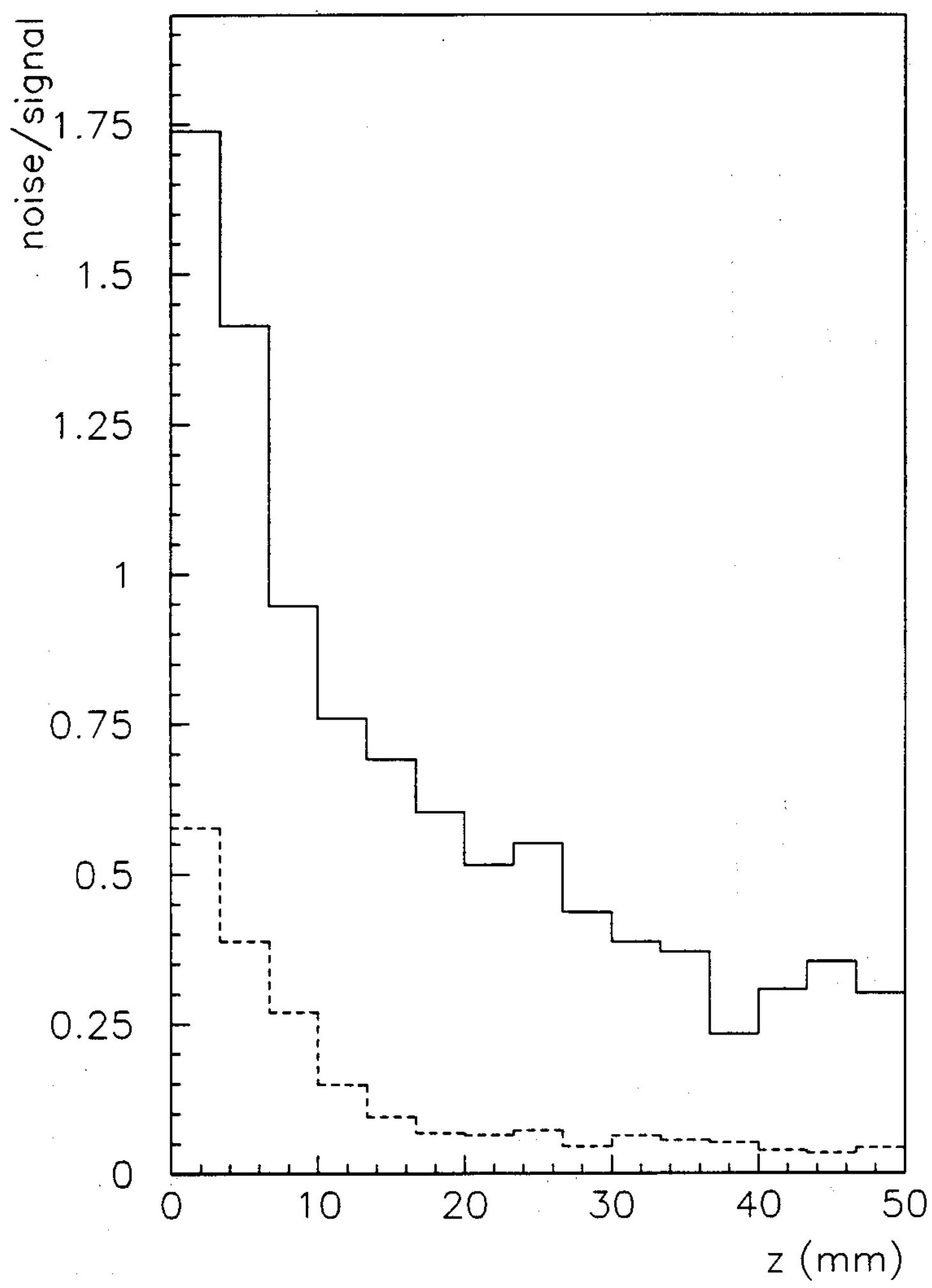

Fig. 5 


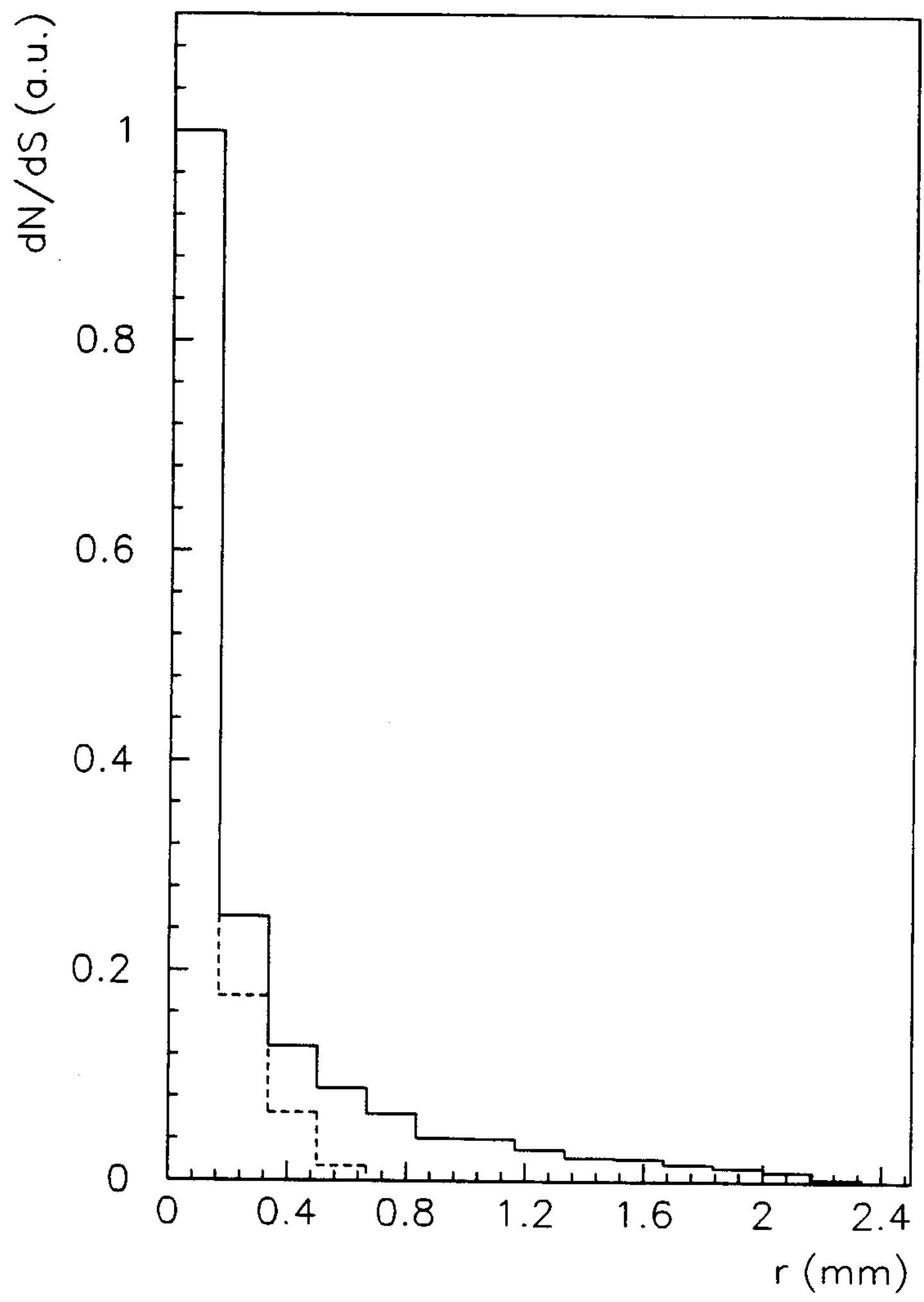

Fig. 6 


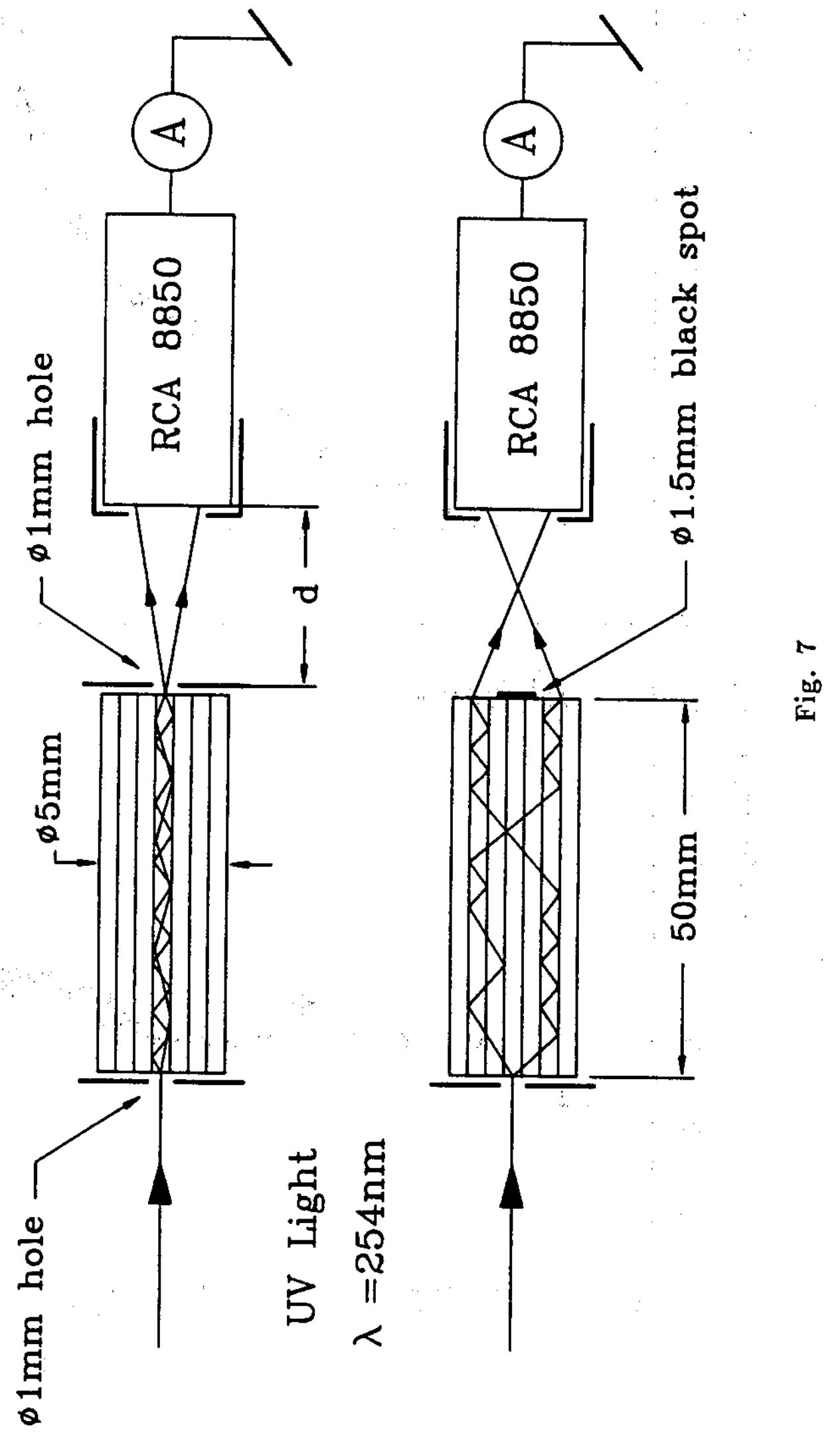



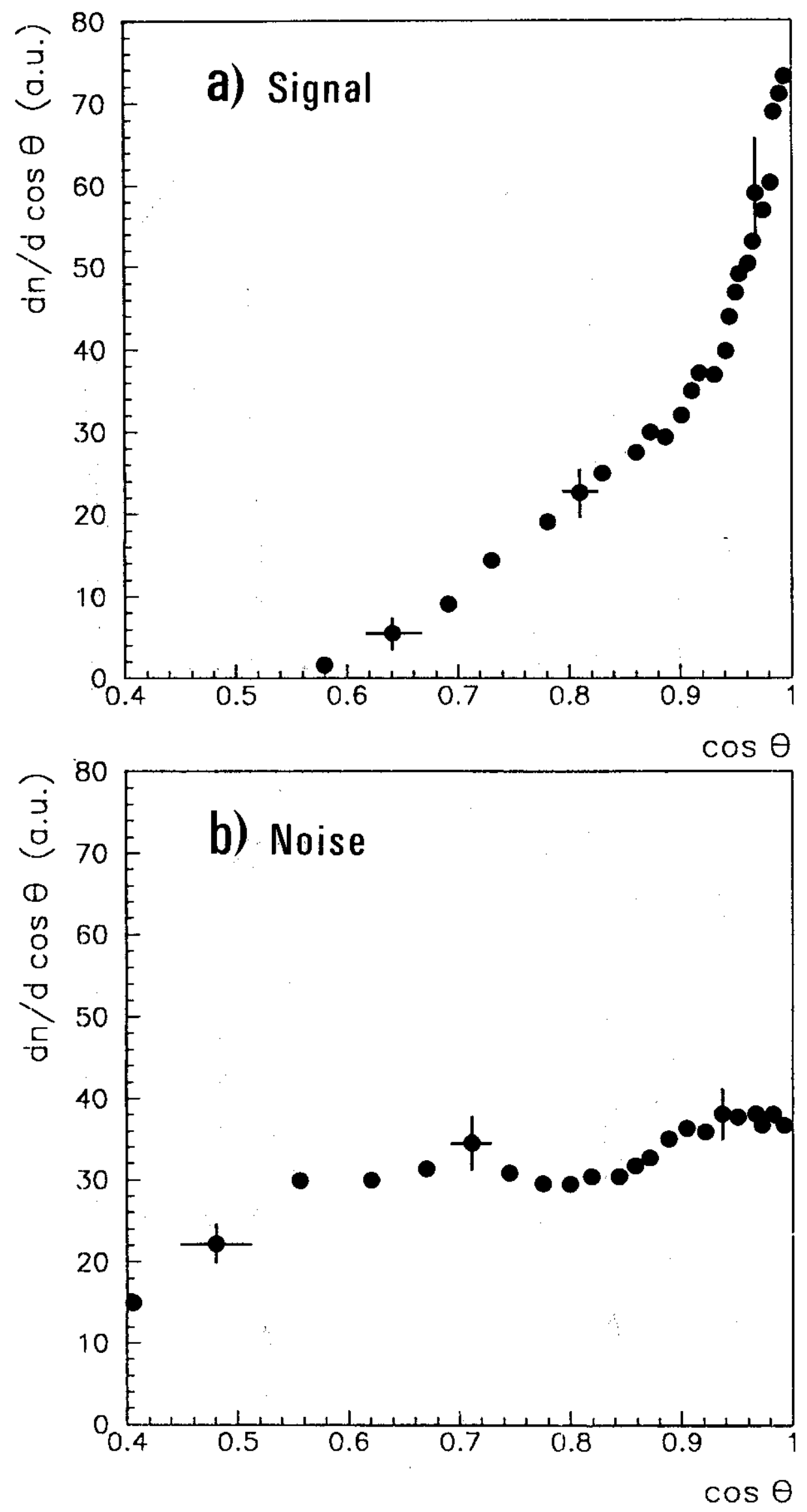

Fig. 8 


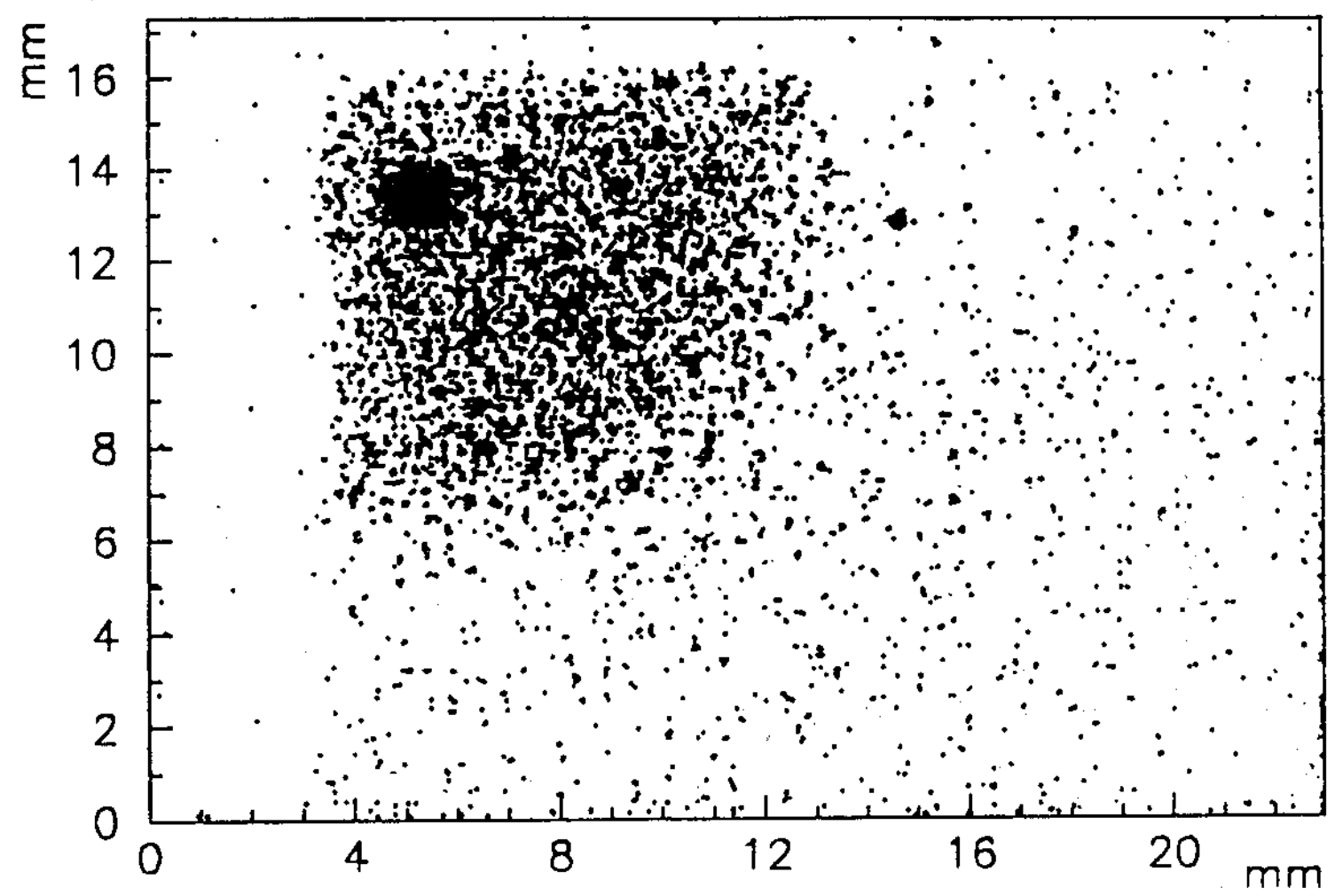

Fig. 9 


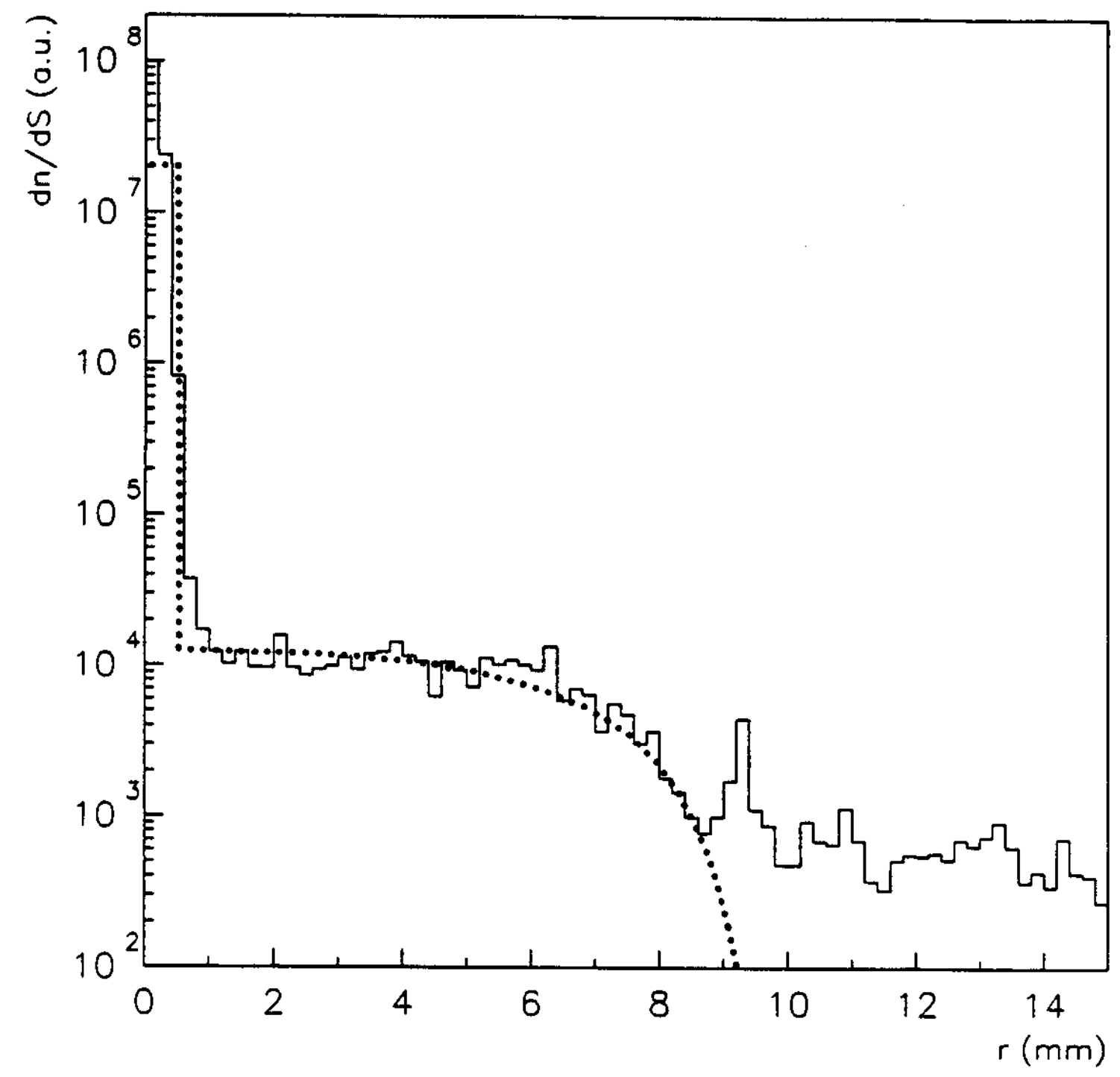

Fig. 10 


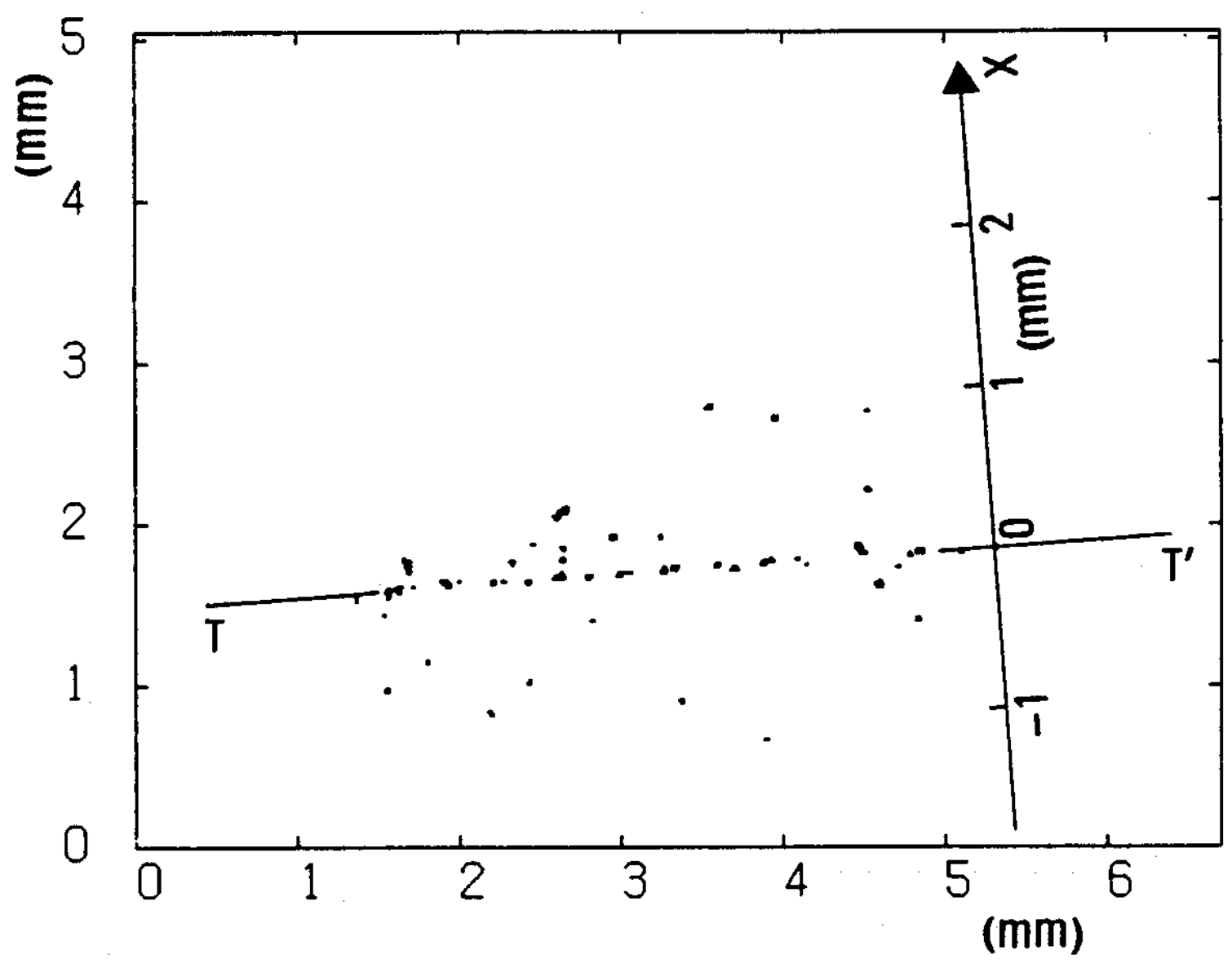

Fig. 11 


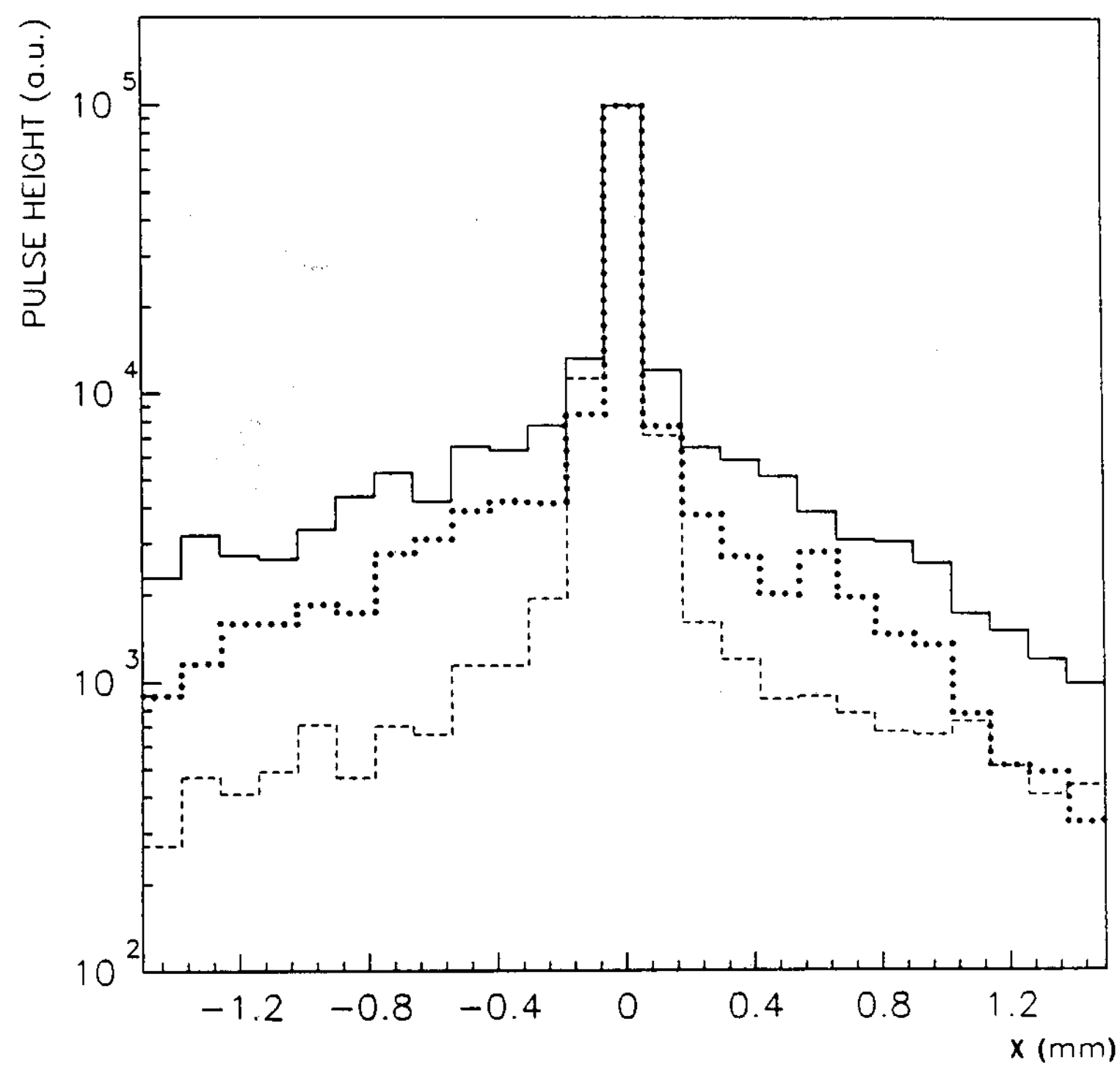

Fig. 12 


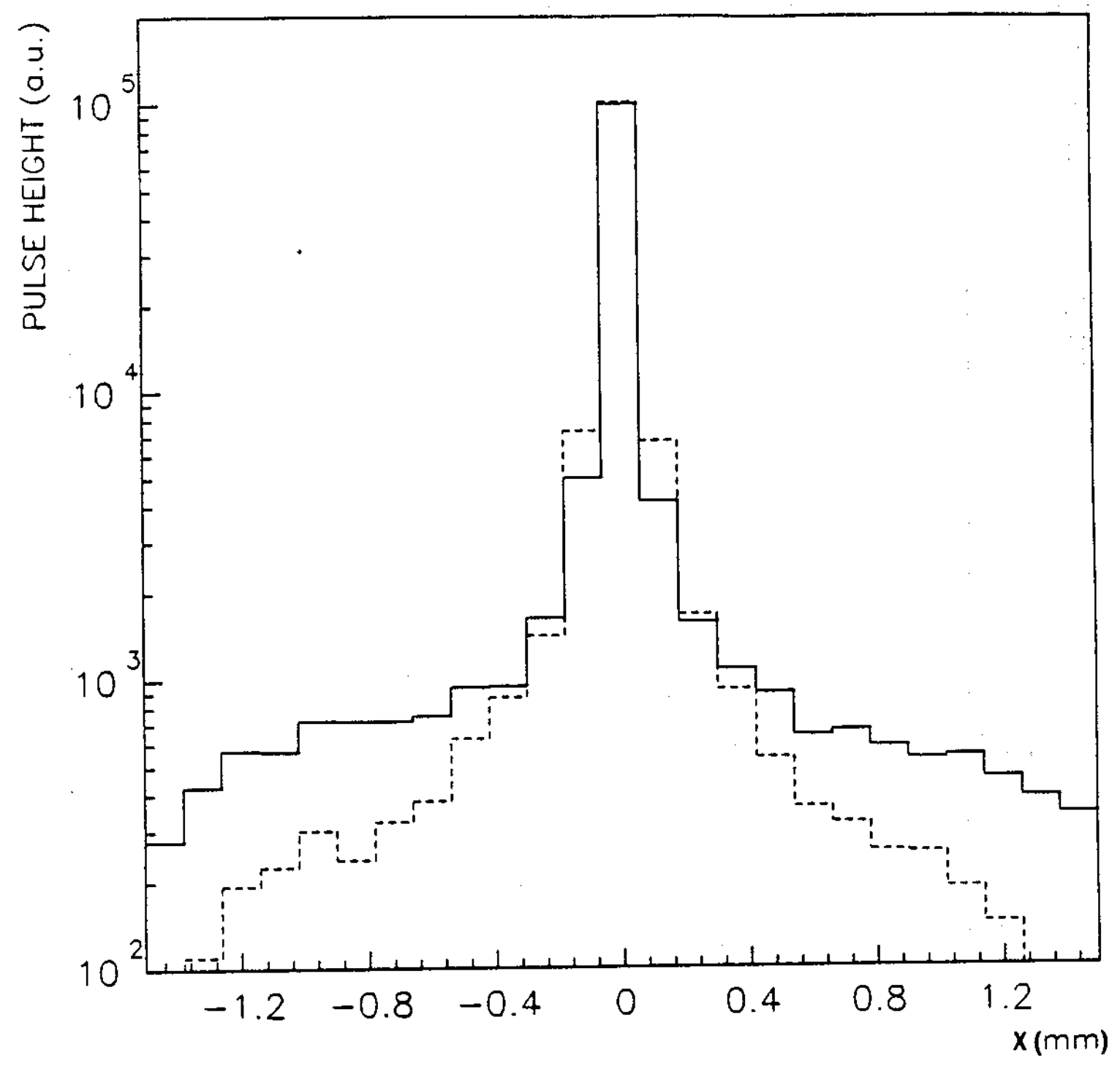

Fig. 13 


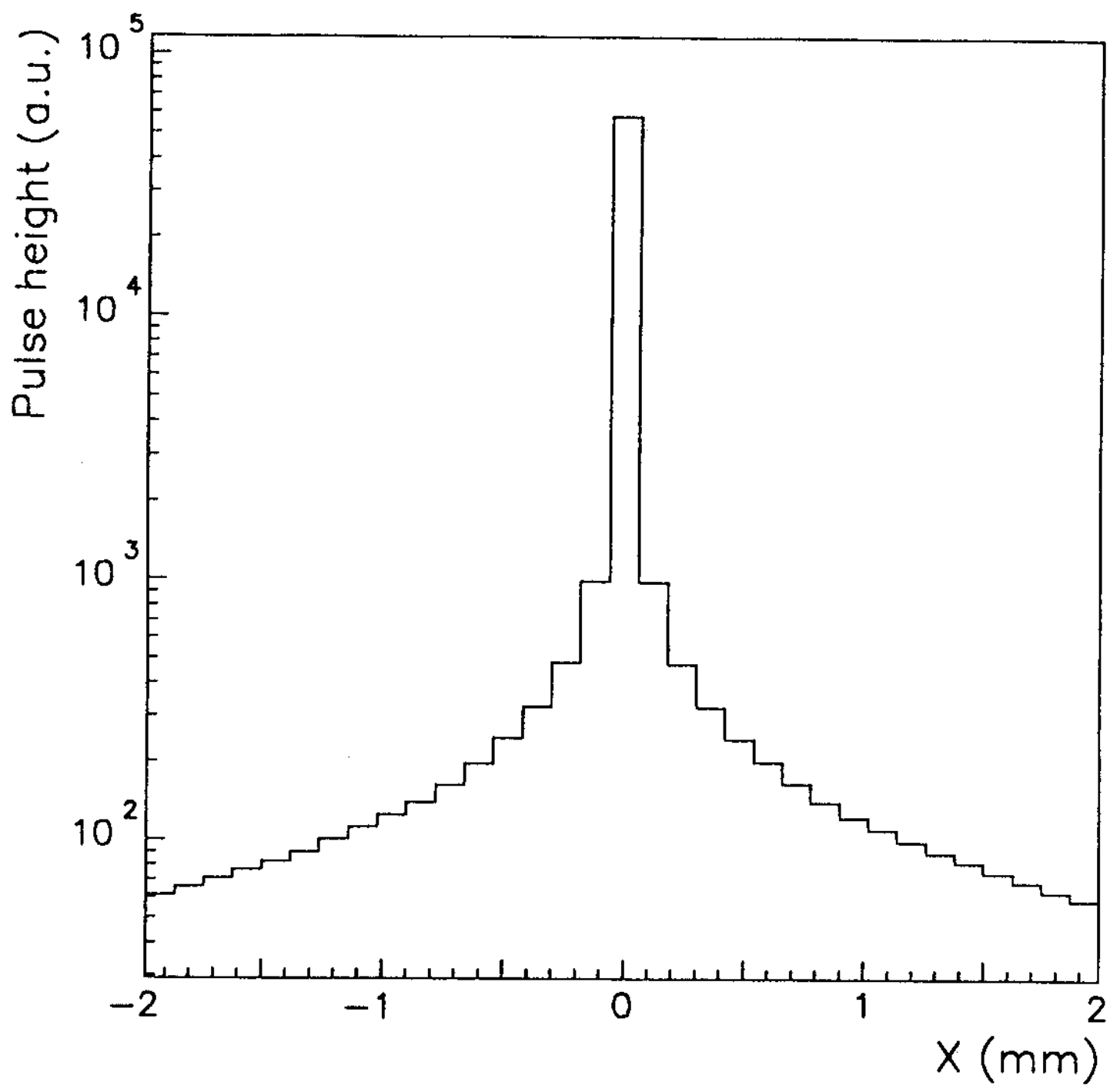

Fig. 14 